\title{
Expression of the forkhead transcription factor FOXL2 correlates with good prognosis in breast cancer patients treated with tamoxifen
}

\author{
PIA WEGMAN ${ }^{1}$, ANNA GÖTHLIN EREMO ${ }^{1}$, ANGELICA LINDLÖF ${ }^{2}$, \\ MATS KARLSSON $^{3}$, OLLE STÅL ${ }^{4}$ and STEN WINGREN ${ }^{1}$ \\ ${ }^{1}$ Department of Clinical Medicine, School of Health and Medical Sciences, Örebro University, 70185 Örebro;
${ }^{2}$ System Biology Research Centre, School of Life Sciences, Skövde University, 54145 Skövde;
${ }^{3}$ Department of Pathology, School of Health and Medical Sciences, Örebro University Hospital,
70185 Örebro; ${ }^{4}$ Department of Clinical and Experimental Medicine/Oncology,
Faculty of Health Sciences, Linköping University, 58185 Linköping, Sweden
}

Received October 13, 2010; Accepted December 3, 2010

DOI: $10.3892 /$ ijo.2011.923

\begin{abstract}
Aromatase is an important enzyme in the local synthesis of oestrogens and its expression has been shown to be increased in breast cancer through the activation of multiple promoters. However, the mechanisms behind this are not yet fully understood. A novel candidate in this context is the transcription factor forkhead box L2 (FOXL2), which has been recognised to be co-expressed with aromatase and transcriptionally active promoter II in developing goat and chicken ovaries. We propose that FOXL2 could be involved in the increased expression of aromatase in breast cancer. We examined FOXL2 and its relation to aromatase in 132 postmenopausal breast cancer patients by immunohistochemistry. Using in silico analysis, we further searched for FOXL2 binding-elements in the aromatase gene promoters. The results demonstrate that FOXL2 is expressed in breast cancer and influences clinical outcome with improved recurrence-free survival in cases with nuclear expression. In a multivariate Cox model, nuclear FOXL2 was a significant prognostic factor in ER-positive patients treated with tamoxifen $(\mathrm{HR}=0.18$, $95 \%$ confidence interval $(\mathrm{CI})=0.04-0.81, \mathrm{P}=0.03)$. Tumours expressing nuclear FOXL2 were also more likely positive for stromal and/or cytoplasmic aromatase $(\mathrm{P}=0.03$ and $\mathrm{P}=0.008$, respectively). In silico analyses revealed binding elements of FOXL2 in promoters I.3, II and I.7 of the aromatase gene of which promoter I.7 was most significant. In conclusion, this is the first study to report that FOXL2 is expressed in breast
\end{abstract}

Correspondence to: Dr Pia Wegman, Department of Clinical Medicine, School of Health and Medical Sciences, Örebro University, 70185 Örebro, Sweden

E-mail: pia.wegman@oru.se

Key words: breast cancer, forkhead box L2, aromatase, tissue specific promoters, in silico cancer and correlates with aromatase as well as with clinical outcome. The results further strengthen a possible binding of FOXL2 to aromatase promoter I.7. Nevertheless, whether FOXL2 is a direct activator of aromatase requires further investigation.

\section{Introduction}

The majority of breast tumours are more or less dependent on oestrogen for their growth, as indicated by the presence of oestrogen-receptors (ERs). After menopause the main production of oestrogen occurs in the peripheral tissues, including the breast, through the enzymatic conversion of androgenic precursors. In breast cancer tissue aromatase is a key enzyme that participates in the aromatisation of androstenedione to oestrone, and testosterone to oestradiol. There have been many studies on the prognostic significance of aromatase in breast cancer, although no conclusive results have been reached $(1,2)$. We have previously found that aromatase is a significant prognostic factor in post-menopausal breast cancer patients, with an improved relapse-free survival in cases with stromal expression (3).

The gene coding for aromatase is regulated through the activation of tissue specific promoters. The normal breast tissue expresses low levels of aromatase primarily via promoter I.4, as opposed to the diseased breast where aromatase is increased through the promoters I.3, II and I.7 (4). The mechanisms behind this promoter shift are not fully understood but are suggested to be a result of differential regulation by hormones, growth factors and cytokines. The factors produced and secreted by breast cancer cells include prostaglandin-E2 $\left(\mathrm{PGE}_{2}\right)$, different cytokines (e.g. IL-6 and IL-11), and tumour necrosis factor- $\alpha$ (TNF $\alpha)$ (5). $\mathrm{PGE}_{2}$ has been recognised to stimulate aromatase activity in surrounding adipose tissue through the induction of the liver-receptor homologue-1 (LRH-1), which is a protein that binds to a nuclear receptor half-site (CAAGGTCA) located 
within promoter II of the aromatase gene (6). The levels of LRH-1 have been shown to be strongly correlated to those of aromatase in breast cancer and have been suggested to be one mechanism by which $\mathrm{PGE}_{2}$ induces aromatase expression in the surrounding adipose tissue (7). Furthermore, the oestrogen related receptor- $\alpha$ (ERR $\alpha)$ has also been demonstrated to be expressed at higher levels in breast cancer. This transcription factor has been proposed to be a positive regulator of aromatase through the activation of promoters I.3 and II $(8,9)$. Another transcription factor of interest in this context is the forkhead box L2 (FOXL2). FOXL2 has been recognised to be co-expressed with aromatase and to transcriptionally activate promoter II of the aromatase gene in developing goat and chicken ovaries $(10,11)$. In addition, during a female to male gender-reversal experiment in chicken embryos it was demonstrated that the inhibition of aromatase lead to a decrease in FOXL2 expression (12). Several members of the FOX family have been shown to be implicated in vertebrate embryogenesis, developmental human disorders and cancer (13-15). To the best of our knowledge FOXL2 has not previously been studied in breast cancer and we therefore aimed to investigate its expression pattern as well as its relationship to aromatase in a cohort of post-menopausal breast cancer patients. The results were compared with clinical and pathological data in order to evaluate its clinical significance. Finally, we performed an in silico search for FOXL2 binding sites in the CYP19A1 promoter to assess the potential of FOXL2 as a transcriptional activator of CYP19A1 gene expression.

\section{Materials and methods}

Patients. The present study included tumour material from 132 post-menopausal patients with stage II or III breast cancer, diagnosed between 1985 and 1994 in the South East Health Care Region of Sweden. Tamoxifen was given post-operatively with a daily dose of $40 \mathrm{mg}$ for 2 or 5 years. Sixty-three patients received tamoxifen for 2 years and 69 patients were given a prolonged treatment for 5 years. The number of recurrences was 27 in the group treated with 2 years of tamoxifen and 26 in the group receiving 5 years of tamoxifen. ER and progesterone-receptor (PgR) content was measured in clinical routine practice by isoelectric focusing before 1988, and later on with enzyme immunoassay (EIA). Samples with concentrations of $\geq 0.1 \mathrm{fmol} / \mu \mathrm{g}$ (or $\geq 0.3 \mathrm{fmol} /$ $\mu \mathrm{g}$ with EIA) were classified as positive. The proportion of ER-positive, PgR-positive and lymph node-positive tumours, was 77,60 and $70 \%$, respectively, and the percentage of large tumours $(>20 \mathrm{~mm})$ was 66 . The mean follow-up time was 9.5 years (range, $0.08-16.9$ years; median, 11 years). The local ethics committee in Linköping, Sweden approved the study with no request of an informed consent.

Cell culture and Western blot analysis. MCF-7 cells were used in Western blot analysis for estimating the basal expression of FOXL2 as well as the specificity of the antibody. Cells were routinely maintained in minimum essential medium (MEM) containing L-glutamine supplemented with $10 \%$ heat inactivated fetal bovine serum and $1 \%$ penicillinstreptomycin $(5000 \mathrm{U} / \mathrm{ml}-5000 \mu \mathrm{g} / \mathrm{ml})$, grown in $75 \mathrm{~cm}^{2}$ flasks at $37^{\circ} \mathrm{C}$ in $5 \% \mathrm{CO}_{2}$ and were passaged when $80 \%$ confluent. Cell culture medium and additives were purchased from Invitrogen (Carlsbad, CA, USA). To isolate proteins MCF-7 cells were washed with PBS before $400 \mu$ RIPA (Igepal Ca630, deoxycholic acid, 10\% sodium dodecyl sulphate, PBS) plus protease inhibitor were added. The mixture was kept on ice for 20 min before being homogenised by flushing through a $0.9 \mathrm{~mm} \varnothing$ syringe ten times. The lysate was centrifuged three times at $15,000 \mathrm{~g}$ for $7 \mathrm{~min}$ and the supernatants were transferred to a new tube after each run. Proteins $(1 \mathrm{mg} / \mathrm{ml})$ were heated in $95^{\circ} \mathrm{C}$ for 5 $\mathrm{min}$ in Laemmli buffer $62.5 \mathrm{mM}$ Tris-HCl, $2 \%$ SDS, $25 \%$ glycerol, $0.01 \%$ bromophenol blue, $5 \% \beta$-mercaptoethanol, $\mathrm{pH}$ 6.8), separated by electrophoresis on a $12 \%$ Tris- $\mathrm{HCl}$ precast gel (Ready Gel ${ }^{\circledR}$, Bio-Rad Laboratories, Hercules, CA, USA) and transferred to a PVDF membrane (Immun-Blot ${ }^{\mathrm{TM}}$ PVDF Membrane, Bio-Rad Laboratories). For all protein detection, ECL Advance ${ }^{\mathrm{TM}}$ Western Blotting Detection Kit (GE Healthcare Amersham, Buckinghamshire, UK) was used. After blocking in ECL-Advance Blocking Agent, the membranes were washed in TBS-Tween and incubated with the primary antibody overnight at $4{ }^{\circ} \mathrm{C}$ (polyclonal anti-mouse/ hamster Fox12 1:5000, AH-diagnostics, Stockholm, Sweden). The membranes were then incubated for $1 \mathrm{~h}$ with secondary HRP conjugated anti-mouse antibody (1:50 000, Santa Cruz Biotechnology, Inc., CA, USA). Proteins were visualised by enhanced chemiluminescence according to directions from the manufacturer. A neutralising peptide for FOXL2 (1:500) served as the negative control (AH-diagnostics, Stockholm, Sweden) whereas $\beta$-tubulin (1:10 000) served as the positive control (Santa Cruz Biotechnology). Precision Plus Protein ${ }^{\mathrm{TM}}$ Standards (Bio-Rad Laboratories) was used as a size marker.

Immunohistochemistry. Morphologically representative areas of formalin-fixed and paraffin-embedded tumours from 132 breast cancer patients were selected and assembled in a tissue microarray. In brief, three $0.8-\mathrm{mm}$ cylindrical cores from each breast cancer specimen were placed in a recipient paraffin block. The tissue microarray block were then cut with a microtome into $4-\mu \mathrm{m}$ thick sections and mounted onto glass slides. The slides were deparaffinised with xylene, rehydrated in decreasing series of ethanol and rinsed in distilled water. Antigen retrieval was accomplished by placing the slides in $10 \mathrm{mM}$ citrate buffer (pH 6.0) and incubated in a $95^{\circ} \mathrm{C}$ water bath for $18 \mathrm{~h}$, followed by washing in PBS-Tween $/ 5 \%$ horse serum at $\mathrm{pH}$ 7.4. After quenching endogenous peroxidase with $3 \% \mathrm{H}_{2} \mathrm{O}_{2}$ in water, the slides were incubated with serum-free protein blocking solution (DakoCytomation, Glostrup, Denmark) for $10 \mathrm{~min}$. The slides were incubated with the primary antibody at $4^{\circ} \mathrm{C}$ overnight (rabbit polyclonal antibody against mouse/hamster FOXL2 1:500 dilution; AH-diagnostics, Stockholm, Sweden). After washing with PBS-Tween $/ 5 \%$ horse serum, the slides were incubated with a secondary antibody (EnVision HRP anti-mouse DAKO) for $30 \mathrm{~min}$. The bound antibody was stained with diaminobenzidine and counterstained with haematoxylin. The slides were finally dehydrated in a series of ethanol and mounted.

Additionally, we used other human tissues to immunohistochemically stain for the protein expression of FOXL2, including appendix, tonsil, liver, brain, striated muscle, skin, lung, thyroid, prostate, placenta, thymus, bone marrow 
Table I. Calculated odds ratios for the likelihood of patients with tumours expressing nuclear FOXL2 to have co-expression of stromal or epithelial aromatase.

\begin{tabular}{|c|c|c|}
\hline $\begin{array}{l}\text { Patients with nuclear } \\
\text { FOXL2 (n) }\end{array}$ & $\begin{array}{c}\text { Patients without nuclear } \\
\text { FOXL2 (n) }\end{array}$ & $\begin{array}{l}\text { Odds ratio } \\
(95 \% \mathrm{CI})\end{array}$ \\
\hline
\end{tabular}

\begin{tabular}{|c|c|c|c|c|}
\hline \multicolumn{5}{|c|}{ Stromal aromatase } \\
\hline Negative & 10 & 18 & 1.0 & \\
\hline Positive & 55 & 39 & $2.54(1.06-6.09)$ & 0.034 \\
\hline \multicolumn{5}{|c|}{ Epithelial aromatase } \\
\hline Negative & 11 & 22 & 1.0 & \\
\hline Positive & 53 & 35 & $3.03(1.31-7.02)$ & 0.008 \\
\hline
\end{tabular}

CI, confidence interval.

and prostate cancer, which were kindly provided from the Department of Pathology at Örebro University Hospital.

Immunohistochemical scoring. Two observers (P.W. and S.W.) first evaluated the slides independently and in cases with different scoring results, a consensus score was reached after re-evaluation. The distribution of immunoreactivity was estimated as the proportion of staining. If $1 / 3$ or more of the tumour cells were stained the section was defined as positive. Staining intensity of the immunoreactions was recorded as 0 , negative; 1 , moderate; and 2 , strong. The proportion and intensity scores were then put together to obtain a total score (proportion of staining + staining intensity). The final categorisation revealed three groups: Negative (total score 0 ), moderate (total score 2), and strong (total score 3 ). Patients were excluded when tissues were non-representative or missing. All immunohistochemical evaluations were performed without knowledge of tumour characteristics and clinical outcome.

Statistical analyses. To examine the relationship between the protein expression and tumour characteristics we used Pearson's Chi-square test. In the Chi-square tests moderate and strong staining for FOXL2 protein expression were grouped together and defined as positive. The test was supplemented with a calculation of odds ratio (OR) for the positive outcome using a logistic regression model with the presence (yes/no) of nuclear FOXL2 as the explanatory factor. The survival curves of recurrences were estimated using the Kaplan-Meier method and the difference between survival rates for patients with different expression levels was assessed by the log-rank test. Furthermore, we performed a multivariate Cox model in order to adjust for the tumour characteristics between the different expression profiles. Differences between groups were judged to be significant with P-values of $<0.05$ and confidence intervals (CIs) were given at the $95 \%$ confidence level. The Statistical Package for Social Sciences (SPSS), version 16.0, was used to perform the statistical analyses.

Bioinformatic analyses. An in silico search for known transcription factor binding sites in the promoter sequence of CYP19A1 was performed using an in-house developed Perl script (16). The consensus of the cis-elements recognised

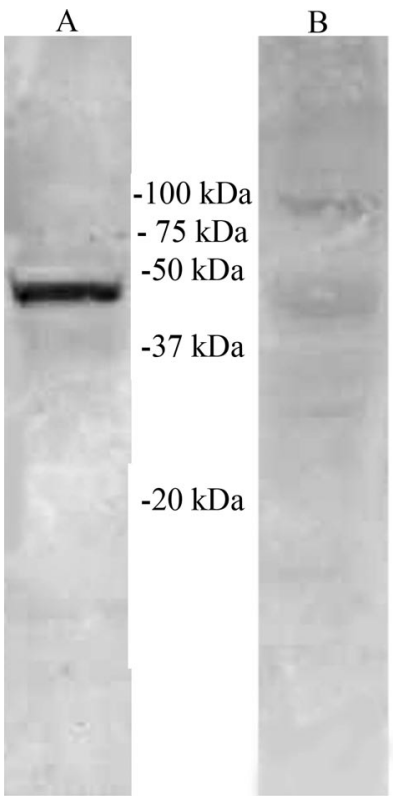

Figure 1. Basal levels of FOXL2 expression in MCF-7 cells detected by Western blot analysis representing the expected size of $45 \mathrm{kDa}$ (A). A neutralising peptide raised against FOXL2 was used as the negative control (B).

by a transcription factor is denoted a motif and can take any character of the IUPAC-code. In this study, two scoring schemes were implemented as search strategies. The first search involved a simple matching approach, where the matching score (Score C) is the number of mismatches to the motif when compared to a subsequence of the promoter. The second search was based on the position weight matrix of the cis-elements and the matching score (Score N) was calculated using the independent scoring scheme described by ZareMirakabad et al (17), i.e., it was assumed that all positions in a given motif are completely independent. The motif consensus and position weight matrix were derived from the study by Benayoun et al (18).

\section{Results}

Expression of FOXL2 in breast tumours. The staining of FOXL2 was informative in $126(95.5 \%)$ of the 132 tumours 
Table II. Nuclear and cytoplasmic expression of FOXL2 in relation to tumour characteristics and tamoxifen duration.

\begin{tabular}{|c|c|c|c|c|c|}
\hline \multirow[b]{2}{*}{ Characteristics } & \multicolumn{5}{|c|}{ Protein expression [n (\%)] } \\
\hline & $\begin{array}{l}\text { FOXL2 } \\
\text { negative }\end{array}$ & $\begin{array}{c}\text { FOXL2 } \\
\text { cytoplasmic }\end{array}$ & $\begin{array}{l}\text { FOXL2 } \\
\text { nuclear }\end{array}$ & $\begin{array}{l}\text { FOXL2 nuclear } \\
\text { and cytoplasmic }\end{array}$ & P-value \\
\hline \multicolumn{6}{|l|}{ Tumour size } \\
\hline$\leq 20 \mathrm{~mm}$ & $3(2.4)$ & $13(10.3)$ & $3(2.4)$ & $24(19.0)$ & 0.35 \\
\hline$>20 \mathrm{~mm}$ & $6(4.8)$ & $38(30.2)$ & $3(2.4)$ & $36(28.6)$ & \\
\hline \multicolumn{6}{|l|}{ Lymph nodes $^{\mathrm{a}}$} \\
\hline Negative & $2(1.6)$ & $17(13.9)$ & $1(0.8)$ & $15(12.3)$ & 0.73 \\
\hline Positive & $7(5.7)$ & $33(27.0)$ & $4(3.3)$ & $43(35.2)$ & \\
\hline \multicolumn{6}{|c|}{ Oestrogen receptor } \\
\hline Negative & $1(0.8)$ & $16(12.7)$ & $1(0.8)$ & $12(9.5)$ & 0.38 \\
\hline Positive & $8(6.3)$ & $35(27.8)$ & $5(4.0)$ & $48(38.1)$ & \\
\hline \multicolumn{6}{|c|}{ Progesterone receptor } \\
\hline Negative & $3(2.4)$ & $27(21.4)$ & $1(0.8)$ & $22(17.5)$ & 0.17 \\
\hline Positive & $6(4.8)$ & $24(19.0)$ & $5(4.0)$ & $38(30.2)$ & \\
\hline \multicolumn{6}{|c|}{ S-phase fraction ${ }^{\mathrm{b}}$} \\
\hline$\leq 10 \%$ & $7(7.1)$ & $25(25.5)$ & $1(1.0)$ & $32(32.7)$ & 0.13 \\
\hline$>10 \%$ & $2(2.0)$ & $19(19.4)$ & $2(2.0)$ & $10(10.2)$ & \\
\hline \multicolumn{6}{|l|}{$\operatorname{ErbB}^{c}$} \\
\hline Negative & $8(6.8)$ & $43(36.4$ & $5(4.2)$ & $49(41.5)$ & 0.74 \\
\hline Positive & $0 \quad(0)$ & $6(5.1)$ & $1(0.8)$ & $6(5.1)$ & \\
\hline \multicolumn{6}{|l|}{ Tamoxifen } \\
\hline 2 years & $2(1.6)$ & $23(18.3)$ & $5(4.0)$ & $30(23.8)$ & 0.13 \\
\hline 5 years & $7(5.6)$ & $28(22.2)$ & $1(0.8)$ & $30(23.8)$ & \\
\hline
\end{tabular}

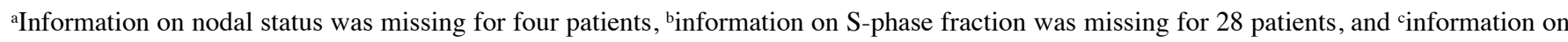
erbB2 status was missing for eight patients.

and the localisation was detected in the nucleus and/or in the cytoplasm of tumour epithelial cells. The immunoreactivity of FOXL2 ranged from negative to moderate and strong expression. Fifty-one patients $(40.5 \%)$ had tumours expressing FOXL2 exclusively in the cytoplasm, whereas 6 cases $(4.8 \%)$ showed only nuclear expression and 9 patients (7.1\%) were FOXL2-negative. Moreover, 60 (47.6\%) patients showed expression of FOXL2 in both cellular compartments. In order to evaluate whether there was a relationship between nuclear and cytoplasmic FOXL2 expression, we performed a correlation test, and the results demonstrated no significant correlation $(\mathrm{RR}=1.07,95 \% \mathrm{CI}=0.94-1.22$, $\mathrm{P}=0.31$ ). In addition, the present patient cohort was previously immunohistochemically stained for aromatase (3) and cases with a tumour expressing nuclear FOXL2 were more likely to have a concomitant expression of aromatase in the stroma and/or in the cytoplasm of cancer epithelial cells $(\mathrm{OR}=2.54,95 \% \mathrm{CI}=1.06-6.09, \mathrm{P}=0.034$; $\mathrm{OR}=3.03$, 95\% CI=1.31-7.02, $\mathrm{P}=0.008$, respectively) (Table I). Using MCF7 cells, we performed Western blot analysis in order to examine the specificity of the antibody as well as the basal level of FOXL2. The level of FOXL2 was relatively high in these cells and the antibody showed a band representing the expected size of $45 \mathrm{kDa}$ (Fig. 1A). Moreover, as the FOXL2 antibody was polyclonal, a neutralising peptide was used as the negative control. The results demonstrated that the peptide was able to quench the signal (Fig. 1B).

We finally defined the protein expression of FOXL2 in separate slides of other human tissues including appendix, tonsil, liver, brain, striated muscle, skin, lung, thyroid, prostate, placenta, thymus, bone marrow and prostate cancer. All tissues were negative for FOXL2 except for prostate cancer, which demonstrated immunoreactivity in the cytoplasm of cancer epithelial cells.

Tumour characteristics and recurrence-free survival. The association between protein expression of FOXL2 and tumour characteristics such as tumour size, lymph node status, ER-status, PgR-status, S-phase fraction, erbB2-status and tamoxifen duration is shown in Table II. No significant correlation to tumour characteristics or tamoxifen duration could be seen for the expression of FOXL2.

Using Kaplan-Meier estimates we then calculated the recurrence-free survival in correlation to FOXL2. Recurrencefree survival was defined as the time from initial surgery to the clinically documented date of local or distant recurrence. In the estimates of the overall population (both ER-positive and ER-negative) we combined patients with tumours having a moderate and strong expression of FOXL2 in the nucleus, independently of cytoplasmic staining, and defined them as 


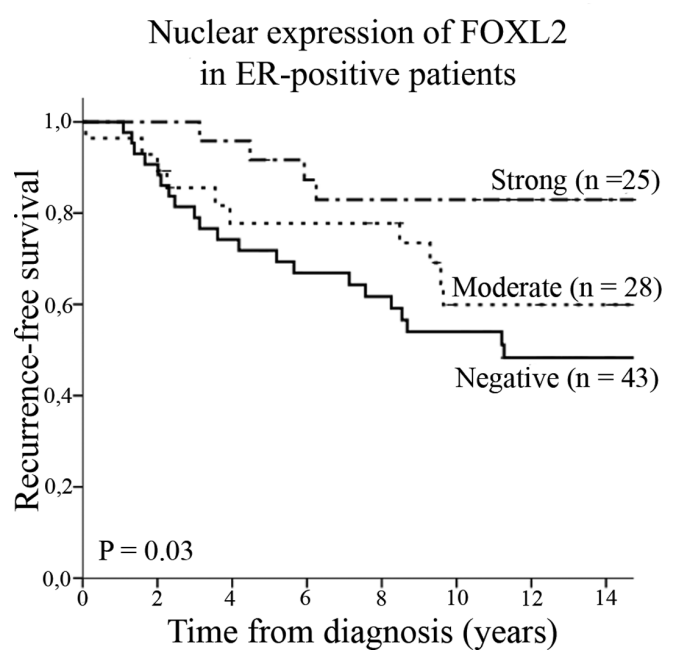

Figure 2. Recurrence-free survival in ER-positive patients according to immunohistochemical staining of nuclear FOXL2. In the group negative for FOXL2, 19 patients received tamoxifen for 2 years and 24 patients for 5 years. Of those with moderate expression, 13 patients received 2 years of tamoxifen and 15 were given 5 years of treatment. Finally, among the patients with strong expression of nuclear FOXL2, 13 patients received 2 years of tamoxifen and 12 patients were treated for 5 years.

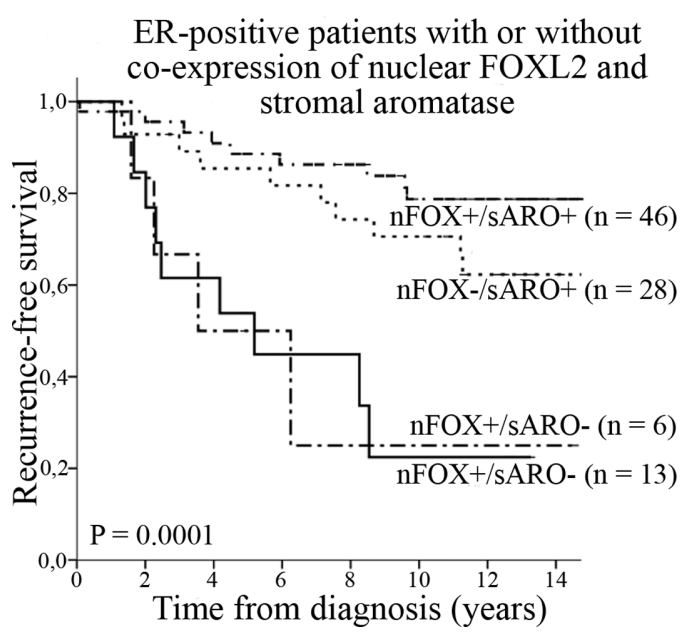

Figure 3. Recurrence-free survival in ER-positive breast cancer patients according to tumours with or without co-expression of nuclear FOXL2 and stromal aromatase.

positive. Patients positive for nuclear FOXL2 demonstrated a significantly improved recurrence-free survival compared to those who were negative $(\mathrm{P}=0.04)$. We then selected ER-positive patients for calculating the recurrence-free survival in relation to the different expression levels of nuclear FOXL2. The results showed a dose-response pattern, with significantly improved recurrence-free survival in patients with increasing levels of FOXL2 ( $\mathrm{P}=0.003$ ) (Fig. 2). This difference could not be seen for the cytoplasmic expression of FOXL2 in the tumours, neither in the total population (ER-positive and ER-negative) nor in the ER-positive cases $(\mathrm{P}=0.61$ and $\mathrm{P}=0.26$, respectively). In a multivariate Cox model (adjusted for tumour size, lymph node status, and S-phase fraction) selecting only ER-positive patients, cases with a tumour expressing high levels of FOXL2 in the nucleus showed a significantly decreased risk of having a

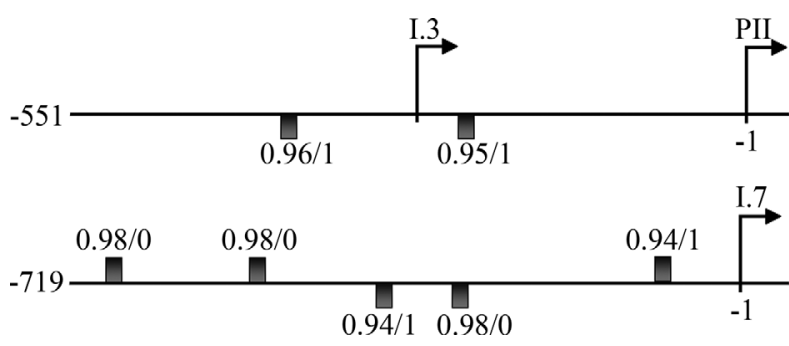

Figure 4. Schematic sequences of aromatase promoter regions I.3/II and I.7 with indicated plausible FOXL2 binding sites. There are two numbers given for each binding site. The first shows the score for the consensus (Score C) search and second for the position weight matrix (Score N) search. Numbers stated below and above the sequence indicate a match in forward and reverse frame, respectively. Each promoter is coupled to a corresponding exon, whose start site is indicated with an arrow (e.g., the start site of exon II is indicated by an arrow and promoter II is located just upstream of this exon).

relapse $(\mathrm{HR}=0.18,95 \% \mathrm{CI}=0.04-0.81, \mathrm{P}=0.03)$. No difference in the risk of relapse could be seen for the cytoplasmic expression of FOXL2.

In addition, the aromatase expression in relation to recurrence-free survival and the risk of relapse has previously been reported in the same patient cohort (3), showing that stromal aromatase is a significant prognostic factor. We therefore included both FOXL2 and aromatase in the multivariate Cox model. The results demonstrated that both nuclear FOXL2 and stromal aromatase were independent prognostic factors in tamoxifen-treated ER-positive cases with a decreased risk of recurrence in those with high expression $(\mathrm{HR}=0.20$, 95\% CI $=0.04-0.96, \mathrm{P}=0.045 ; \mathrm{HR}=0.32,95 \%$ CI 0.10-0.99, $\mathrm{P}=0.048$, respectively). Finally, we compared the recurrencefree survival in relation to the co-expression of nuclear FOXL2 and stromal aromatase, which revealed that patients with a concomitant expression of FOXL2 and aromatase had a significantly better disease-free survival compared to cases with other expression patterns $(\mathrm{P}=0.0001)$ (Fig. 3).

FOXL2 binding sites in CYP19A1 promoters. In order to assess whether FOXL2 could plausibly bind to and thereby activate CYP19A1, we performed an in silico search analysis of the I.3/ II and I.7 promoters of CYP19Al using the motif and weight matrix of the FOXL2 response element (FLRE) identified by Benayoun et al (18). The promoter sequences were extracted from previously published studies $(19,20)$, whereas the motif and weight matrix were derived from the study by Benayoun et al (18). We searched with both the motif and the position weight matrix against the promoter sequences using commonly applied scoring schemes. The search revealed seven plausible binding sites with a maximum of one mismatch against the motif. Two of them were located in promoter I.3/II and five in I.7 (Fig. 4). Of the identified sites, three were perfect matches to the motif and these were all located in promoter I.7. The binding sites identified in I.3/II contained both one mismatch and, additionally, one of those was located within the exon of promoter I.3.

\section{Discussion}

FOXL2 has been identified as the earliest known marker for ovarian differentiation in mammals with an association to 
the blepharophimosis-ptosis-epicanthus inversus syndrome (BPES) where genetic mutations of different origin give rise to premature ovarian failure and/or cranio-facial malformation (21-23). The implication of FOXL2 in cancer has not been widely studied although Kalfa et al (24) have reported a decreased expression of FOXL2 in juvenile ovarian granulosa cell tumours with an aggressive pattern of progression. Additionally, Benayoun et al (25) recently proposed that FOXL2 could act as a tumour suppressor, at least in ovarian granulosa cell tumours.

To the best of our knowledge, this is the first study to report that FOXL2 is expressed in breast cancer and moreover, that it correlates with both stromal and cancer epithelial aromatase as well as with clinical outcome. Patients with tumours expressing nuclear FOXL2 had a significant improved prognosis compared to those lacking nuclear FOXL2. In the same patient cohort, we previously reported that cases with a tumour expressing stromal aromatase had an improved relapse-free survival (3). To explore whether there was a correlation between the expression of FOXL2 and aromatase in this cohort, we combined the expression pattern of these proteins and found that patients with FOXL2 in the nucleus more likely expressed aromatase in the cytoplasm of cancer epithelial cells and/or in the stroma. These results strengthen previous reports of a relationship between FOXL2 and aromatase that has been shown in the gonads of goat foetuses, chicken, rainbow trout and Nile Tilapia $(10,11,26,27)$.

The cellular localisation of FOXL2 staining in the breast cancer specimens demonstrated a heterogeneous pattern, ranging from cytoplasmic to nuclear or both. The expectation of a transcription factor is that it should be active/expressed in the nucleus. However, differences in localisation could be due to post-translational modifications or a consequence of genetic alterations/mutations. However, the latter has not yet been shown for FOXL2 in breast cancer. Post-translational modifications determine the cellular localisation and activity of FOX factors. In general, when FOX proteins are located in the nucleus they have been suggested to act as transcription factors, whereas cytoplasmic FOX proteins have been proposed to be inactive and subjected to proteasomal degradation (13). Whether FOXL2 undergoes post-translational modification has been a matter of debate, although Benayoun et al $(28,29)$ have reported that FOXL2 is highly modified by phosphorylation, acetylation and sumoylation, which can alter the activity of the protein.

For the immunohistochemical detection of FOXL2 in breast cancer specimens as well as in Western blot analysis, we used a polyclonal antibody raised against mouse/hamster FOXL2. The homology between mouse and human FOXL2 is $92 \%$, and the antibody used included a slight difference of two amino acids between human and mouse. However, before using the antibody a Protein BLAST was performed, which showed a high correspondence to FOXL2 in different species, including humans. We also investigated the specificity of the FOXL2 antibody in MCF-7 cells by Western blot analysis. The results demonstrated a strong signal of FOXL2 with a distinct band at an expected size, which supports the specific binding of the antibody. To further ensure the specificity in our human system we used a neutralising peptide showing that the signal of FOXL2 was quenched.

Furthermore, Batista et al (30) investigated potential pathways transcriptionally modulated by FOXL2 in a cellular model of ovarian granulosa cells. Their results pointed towards a multifunction of this particular factor including the regulation of genes involved in cholesterol metabolism, steroid aromatisation, regulation of apoptosis, and regulation of pro-inflammatory factors. A number of these genes were stimulated by FOXL2 whereas others were suppressed. Two of the factors stimulated by FOXL2 were cyclooxygenase-2 (2) and LRH-1, which both have shown a connection to an increased transcriptional activation of the CYP19A1 promoters I.3 and II in breast cancer. Additionally, in a gonadal gender-reversal experiment Uhlenhaut et al (31) performed a genome-wide expression profiling of wild-type ovaries, reprogrammed gender-reversed ovaries and testes in mice. They reported that when FOXL2 was deleted, CYP19A1 was the most significantly down-regulated gene, which further strengthens the potential association between FOXL2 and CYP19A1. Our in silico search analysis revealed a number of possible binding sites for FOXL2 in the promoters I.3/II as well as I.7. According to the results, I.7 is a more interesting target than I.3/II as three perfect matches and two nearly perfect matches (only 1 mismatch against the consensus) of the FLRE were found in this promoter as opposed to only two nearly perfect matches in I.3/II. Benayoun et al (17) found that the number of FLREs in the promoter reflects the amplitude of the response, as an artificial luciferase promoter reporter containing four FLRE sequences has significantly higher expression levels than a promoter containing only two FLREs. Moreover, they concluded that the number of identified FLREs is significantly higher in the promoter sequences of FOXL2responsive genes compared to all the promoter sequences in the remaining genome. This is of interest, as there has been no report of FOXL2 binding to promoter I.7 in humans. Therefore, the motif search strengthens the hypothesis of a connection between FOXL2 and CYP19A1 gene expression, but whether CYP19A1 is a direct target of FOXL2 in breast cancer remains to be clarified.

In conclusion, this is the first study to show that the forkhead transcription factor FOXL2 is expressed in breast cancer and is associated with the expression of aromatase as well as with clinical outcome. The significant binding possibilities of FOXL2 to aromatase promoter I.7, and to a lesser extent the promoters I.3 and II, further support a connection between FOXL2 and aromatase. Nevertheless, the results need to be confirmed in a larger cohort and further evaluated in functional studies.

\section{Acknowledgements}

We thank professor Lennart Bodin (Swedish Business School, Örebro University) for support in parts of the statistical analyses. This study was supported by grants from the Swedish Cancer Foundation, Swedish Cancer and Allergy Society, Magnus Bergvall Cancer Foundation, and Percy Falk Cancer Foundation. 


\section{References}

1. Zhang Z, Yamashita H, Toyama T, et al: Quantitative determination, by real-time reverse transcription polymerase chain reaction, of aromatase mRNA in invasive ductal carcinoma of the breast. Breast Cancer Res 5: R250-R256, 2003.

2. Salhab M, Reed MJ, Al Sarakbi W, Jiang WG and Mokbel K: The role of aromatase and 17-beta-hydroxysteroid dehydrogenase type $1 \mathrm{mRNA}$ expression in predicting the clinical outcome of human breast cancer. Breast Cancer Res Treat 99: 155-162, 2006.

3. Licznerska BE, Wegman PP, Nordenskjöld B and Wingren S: In situ levels of oestrogen producing enzymes and its prognostic significance in postmenopausal breast cancer patients. Breast Cancer Res Treat 112: 15-23, 2008.

4. Bulun SE, Chen D, Lu M, et al: Aromatase excess in cancer of breast, endometrium and ovary. J Steroid Biochem Mol Biol 106 81-96, 2007.

5. Simpson ER: Biology of aromatase in the mammary gland. J Mammary Gland Biol Neopl 5: 251-258, 2000.

6. Clyne CD, Speed CJ, Zhou J and Simpson ER: Liver receptor homologue-1 (LRH-1) regulates expression of aromatase in preadipocytes. J Biol Chem 277: 20591-20597, 2002.

7. Zhou J, Suzuki T, Kovacic A, et al: Interactions between prostaglandin E2, Liver receptor homologue-1, and aromatase in breast cancer. Cancer Res 65: 657-663, 2005.

8. Yang C, Zhou D and Chen S: Modulation of aromatase expression in the breast tissue by ERR $\alpha-1$ orphan receptor. Cancer Res 58: 5695-5700, 1998.

9. Chen S, Itoh T, Wu K, Zhou D and Yang C: Transcriptional regulation of aromatase expression in human breast tissue. J Steroid Biochem Mol Biol 83: 93-99, 2003.

10. Pannetier M, Fabre S, Batista F, et al: FOXL2 activates P450 aromatase gene transcription: towards a better characterization of the early steps of mammalian ovarian development. J Mol Endocrinol 36: 399-413, 2006.

11. Govoroun MS, Pannetier M, Pailhoux E, et al: Isolation of chicken homolog of the FOXL2 gene and comparison of its expression pattern with those of aromatase during ovarian development. Dev Dyn 231: 859-870, 2004.

12. Hudson QJ, Smith CA and Sinclair AH: Aromatase inhibition reduces expression of FOXL2 in the embryonic chicken ovary. Dev Dyn 233: 1052-1055, 2005.

13. Myatt SS and Lam EW: The emerging roles of forkhead box (FOX) proteins in cancer. Nat Rev Cancer 7: 847-859, 2007.

14. Hannenalli S and Kaestner KH: The evolution of FOX genes and their role in development and disease. Nat Rev Cancer 10: 233-240, 2002

15. Carlsson P and Mahlapuu M: Forkhead transcription factors: Key players in development and metabolism. Dev Biol 250: 1-23, 2002.

16. Schwartz R, Phoenix T and Foy DB: Learning Perl. 5th edition. O'Really Media, USA, 2008.
17. Zare-Mirakabad F, Ahrabian H, Sadeghi M, Nowzari-Dalini A and Goliaei B: New scoring scheme for finding motifs in DNA sequences. BMC Bioinformatics 10: 93-113, 2009.

18. Benayoun BA, Caburet S, Dipietromaria A, et al: The identification and characterization of a FOXL2 response element provides insights into the pathogenesis of mutant alleles. Hum Mol Genet 17: 3118-3127, 2008.

19. Chen D, Reierstad S, Lu M, Lin Z, Ishikawa H and Bulun SE: Regulation of breast cancer-associated aromatase promoters. Cancer Lett 273: 15-26, 2009.

20. Sebastian S, Takayama K, Shozu M and Bulun SE: Cloning and characterization of a novel endothelial promoter of the human CYP19 (aromatase P450) gene that is up-regulated in breast cancer tissue. Mol Endocrinol 16: 2243-2254, 2002.

21. Cocquet J, Pailhoux E, Jaubert F, et al: Evolution and expression of FOXL2. J Med Genet 39: 916-922, 2002.

22. Zlotogora J, Sagi M and Cohen T: The blepharophimosis, ptosis, and epicanthus inversus syndrome: delineation of two types. Am J Hum Genet 35: 1020-1027, 1983.

23. Crisponi L, Deiana M, Loi A, et al: The putative forkhead transcription factor FOXL2 is mutated in blepharophimosis/ ptosis/epicanthus inversus syndrome. Nature Genet 27: 159-165, 2001.

24. Kalfa N, Philibert P, Patte C, et al: Extinction of FOXL2 expression in aggressive ovarian granulosa cell tumors in children. Fertility Sterility 87: 896-901, 2007.

25. Benayoun BA, Kalfa N, Sultan C and Veitia RA: The forkhead factor FOXL2: A novel tumor suppressor? Biochim Biophys Acta 1805: 1-5, 2010

26. Baron D, Cocquet J, Xia X, Fellous M, Guiguen Y and Veitia RA An evolutionary and functional analysis of Fox 12 in rainbow trout gonad differentiation. J Mol Endocrinol 33: 705-715, 2004.

27. Wang DS, Kobayashi T, Zhou LY, et al: Foxl2 up-regulates aromatase gene transcription in a female-specific manner by binding to the promoter as well as interacting with Ad4 binding protein/steroidogenic factor 1. Mol Endocrinol 21: 712-725, 2007.

28. Benayoun BA, Auer J, Caburet S and Veitia R: The post-translational modification profile of the forkhead transcription factor FOXL2 suggests the existence of parallel processive/concerted modification pathways. Proteomics 8: 3118-3123, 2008.

29. Benayoun BA, Batista F, Auer J, Dipietromaria A, L'Hôte D, De Baere E and Veitia RA: Positive and negative feedback regulates the transcription factor FOXL2 in response to cell stress: evidence for a regulatory imbalance induced by diseasecausing mutations. Hum Mol Genet 18: 632-644, 2009.

30. Batista F, Vaiman D, Dausset J, Fellous M and Veitia RA: Potential targets of FOXL2, a transcription factor involved in craniofacial and follicular development, identified by transcriptomics. Proc Natl Acad Sci 104: 3330-3335, 2007.

31. Uhlenhaut NH, Jakob S, Anlag K, et al: Somatic sex reprogramming of adult ovaries to testes by FOXL2 ablation. Cell 139: $1130-1142,2009$ 